\title{
Ureteral Stent Placement Using a Double J-Catheter in the Treatment of Ectopic Ureter in a Dog
}

\author{
Guilherme Sembenelli, Jose Sergio Costa Junior, Monica Carolina Nery Wittmaack, Gabriel Luiz Montanhim, \\ Luis Gustavo Gosuen Gonçalves Dias \& Paola Castro Moraes
}

\begin{abstract}
Background: Ectopic ureter is a congenital anomaly in which the final segment of one or both ureteral orifices is located distal to the bladder trigone. It may be classified as intra- or extramural. Surgical treatment of ectopic ureters in dogs is recommended and the approach varies with the classification. In the postoperative period, complications are common. When stenosis of the new ureteral ostium occurs, immediate repeated surgery is recommended. This study aimed at using the double $\mathrm{J}$ catheter placement following neoureterostomy to treat urethral obstruction secondary to the surgical treatment of an intramural ectopic ureter in a dog.

Case: An 8-month-old female French bulldog with dysuria and urinary incontinence was seen at a private veterinary hospital in Jaboticabal, São Paulo. The patient had previously been diagnosed with an intramural ectopic ureter on the right side following imaging tests (ultrasound, computed tomography, and abdominal radiography, excretory urography) and had undergone neoureterostomy and closure of the intramural pathway approximately a year ago. Ultrasonographic examination showed dilation of the caudal portion of the ureter and hydroureter, which was suggestive of right ureteral stenosis. Computed tomography was also performed to evaluate the kidneys, ureters, and bladder; an increase in the diameter of the right ureter in its middle portion and close to the bladder triangle was observed. A new surgical intervention was indicated and performed. The ureteral route was identified in a region of the bladder trigone, incised, and probed with a urethral probe No. 04. The intramural course in the proximal urethra was identified and probed with a 16G epidural catheter. It was necessary to perform a neoureterostomy. A longitudinal incision (spatulation) of approximately $5 \mathrm{~mm}$ was made in the distal portion of the right ureter to increase the circumference of the anastomosis. The double J 4.7 French (Fr) catheter was inserted through the new ureter ostium into the bladder and advanced into the right kidney in a retrograde manner. Once the proximal end of the double $J$ catheter reached the renal pelvis, the guidewire was withdrawn slowly to allow the catheter to bend in the areas of the renal pelvis and the trigone. The distal end of the double $\mathrm{J}$ catheter that extended beyond the bladder lumen was sectioned for better bladder closure. The patient underwent clinical evaluation and laboratory tests (complete blood count and serum creatinine concentration, urine test with bacteriological culture and susceptibility test) 2 weeks after the procedure and, subsequently, every 3 months. Ultrasonography of the urinary tract was performed every 2 months.

Discussion: We used a double $\mathrm{J}$ catheter in the patient due to a previous obstruction of the ureter ostium after the first surgical procedure. In this way, complications such as postoperative obstructions due to ureteritis and ureteral constriction were avoided and ureteral anastomosis was facilitated. It has been reported that animals subjected to ureteral stent placement have high incidences of dysuria and urinary tract infection, and low incidences of stent migration and occlusion. In this case, no signs of occlusion or obstruction of the implant were identified, but there was a recurrence of urinary tract infections. These frequently cause urethral obstruction associated with the healing of the new ureteral ostium. Patient follow-up and findings associated with the long-term insertion of the double $\mathrm{J}$ catheter provide support for the clinical relevance of the present report.
\end{abstract}

Keywords: double J catheter, discoespondilitis, ectopic ureter, neoureterostomy. 


\section{INTRODUCTION}

Surgical treatment of dogs with ectopic ureter is recommended; the approach varies with the classification of ectopic ureter [6,8]. For intramural ectopic ureters, neoureterostomy associated with the closure of the intramural pathway is generally preferred; it yields good outcomes [8]. In the postoperative period, complications such as dysuria, polyuria, and hematuria are common; in more severe cases, uroabdomen may occur. When stenosis of the new ureteral ostium occurs, immediate surgical reintervention is recommended to prevent the formation of hydroureter and hydronephrosis. Bacterial cystitis is also observed in the short and long term after the surgical procedure [8].

The double $\mathrm{J}$ catheter (ureteral stent) has been used in veterinary medicine research for the treatment of malignant ureteral obstruction [1], obstructive pyonephrosis [4], congenital bilateral ureteral stenosis [5], and benign ureteral obstruction [9]. However, there is no account in the literature on the use of the double $\mathrm{J}$ catheter in dogs with ectopic ureters. In this report, the surgical technique of neoureterostomy followed by implantation of a double-J catheter is described, highlighting its possible advantages and disadvantages in the treatment of ectopic ureter in dogs. The long-term follow-up of a patient with a double-J catheter with recurrent bacterial cystitis associated with discoespondilitis is also elaborated. This study aimed to use the double $\mathrm{J}$ catheter following neoureterostomy in urethral obstruction secondary to the surgical treatment of an intramural ectopic ureter in a dog.

\section{CASE}

An 8-month-old female French bulldog with dysuria and urinary incontinence was seen at a private veterinary hospital in Jaboticabal in São Paulo. The patient had been previously diagnosed with an intramural ectopic ureter on the right side following imaging tests (ultrasound, computed tomography, and abdominal radiography, excretory urography) and had undergone neoureterostomy and closure of the intramural pathway approximately a year ago.

At the time of treatment, the patient underwent physical and laboratory tests (blood count, renal and hepatic biochemical profile, urinalysis) and the only abnormality detected was the creatinine concentration $(1.8 \mathrm{mg} / \mathrm{dL})$. Ultrasonographic examination showed dilation of the caudal portion of the ureter and hydrou- reter, which was suggestive of right ureteral stenosis. Computed tomography was also performed to evaluate the kidneys, ureters, and bladder. An increase in the diameters of the right ureter in its middle portion (4.4 $\times 5.5 \mathrm{~mm}$ ) [Figure $1 \mathrm{~A}$ ] and close to the bladder triangle $(8.4 \times 5.9 \mathrm{~mm})$ were observed (Figure 1B). There was a minimal passage of contrast through the intramural path with no contrast leakage to the bladder lumen through the right ureteral ostium (Figure 1C). Through the three-dimensional reconstruction of computed urotomography, the dilation of the ureter in the region of the bladder trigone and the passage of contrast through the right intramural path were visualized (Figure 2).

A new surgical intervention was indicated and performed. Cystotomy was performed on the dorsal face of the bladder. During the internal exploration of the bladder, obstruction of the ureteral path and non-occlusion of the intramural path of the ectopic ureter were observed. The urethral pathway was identified in a region of the bladder trigone, incised, and probed with a urethral probe No. 04 ${ }^{1}$. The intramural pathway in the proximal urethra was identified and probed with a 16 $\mathrm{G}$ epidural catheter ${ }^{2}$ (Figure 3A). A neoureterostomy was required. A longitudinal incision (spatulation) of approximately $5 \mathrm{~mm}$ was made in the distal portion of the right ureter to increase the circumference of the anastomosis. The first suturing was performed at the apex of the spatulated incision using a 4-0 monofilament synthetic absorbable $\left(\text { Caprofyl }{ }^{\circledR}\right)^{3}$ thread. The remainder of the anastomosis, with simple interrupted stitches between the bladder and the ureteral mucosae and extended to the entire ureteral edge, was well-positioned inside the organ lumen (Figure 3B).

The J 4.7 Fr double catheter (Plast-med $\left.{ }^{\circledR}\right)^{4}$ was inserted into the new ureteral ostium in the bladder and advanced into the right kidney in a retrograde manner. Once the proximal end of the double $\mathrm{J}$ catheter reached the renal pelvis, the guidewire was withdrawn slowly to allow the catheter to bend in the areas of the renal pelvis and the trigone. The distal end of the double-J catheter that extended beyond the bladder lumen was sectioned for better bladder closure (Figure 3C). The intramural ureter pathway in the proximal urethra was occluded on the dorsal face of the bladder. A 4-0 nylon ${ }^{5}$ was used to place 2 simple points that were separated towards the serous layer and the mucous layer, surrounded the probe, and anchored the ureteral mucosa; there was 

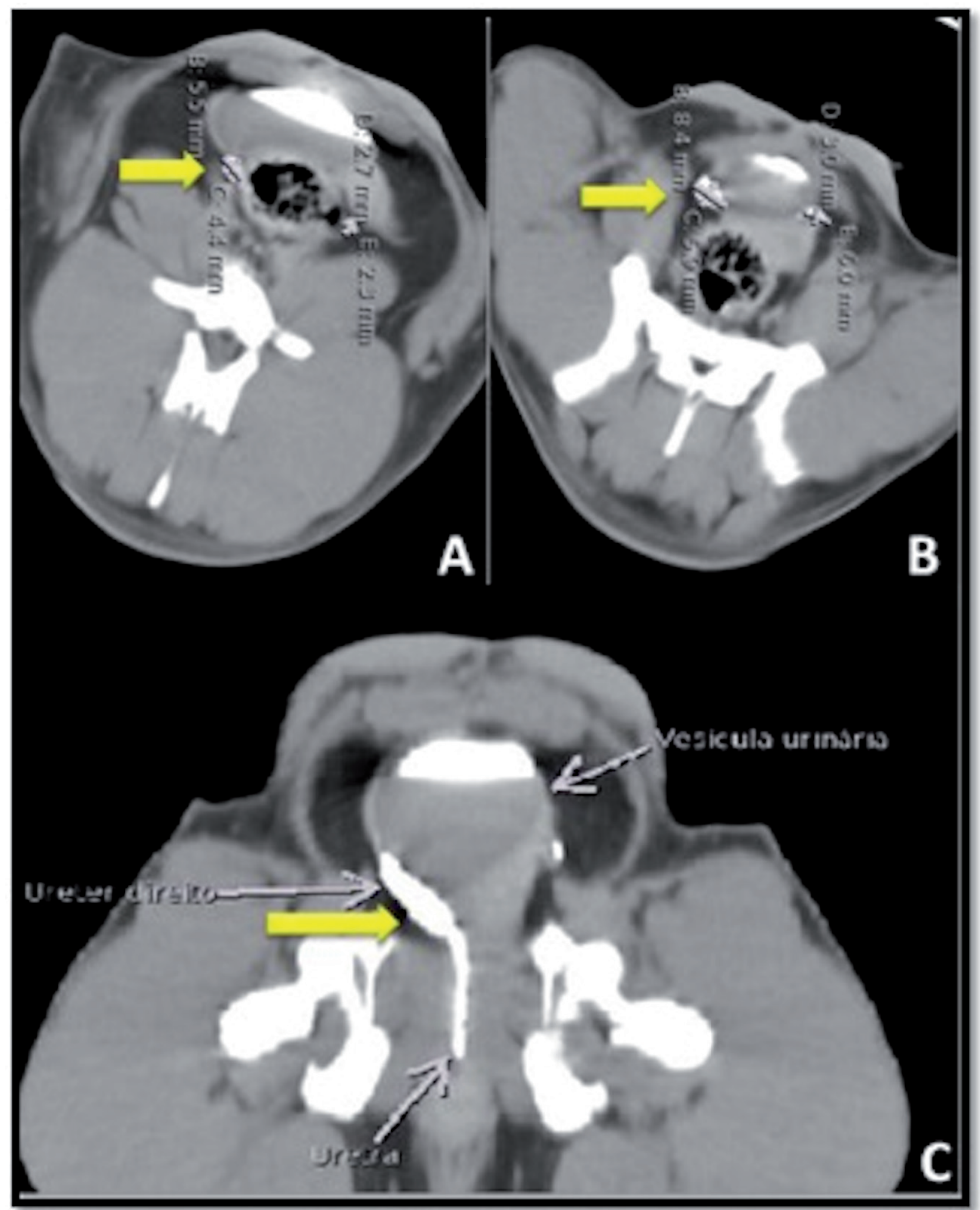

Figure 1. Computed tomography image showing an increase in the diameter of the right ureter. A- Middle ureter. B- Distal ureter. C- Intramural path of the dilated right ureter (arrow). 
no penetration of the lumen of the bladder (Figure 3D). The number 10 ureteral tube was removed after occluding sutures on the outer face of the bladder were completed, ensuring that the diameter of the urethra was not compromised. Finally, a caprofyl@ ${ }^{3}$

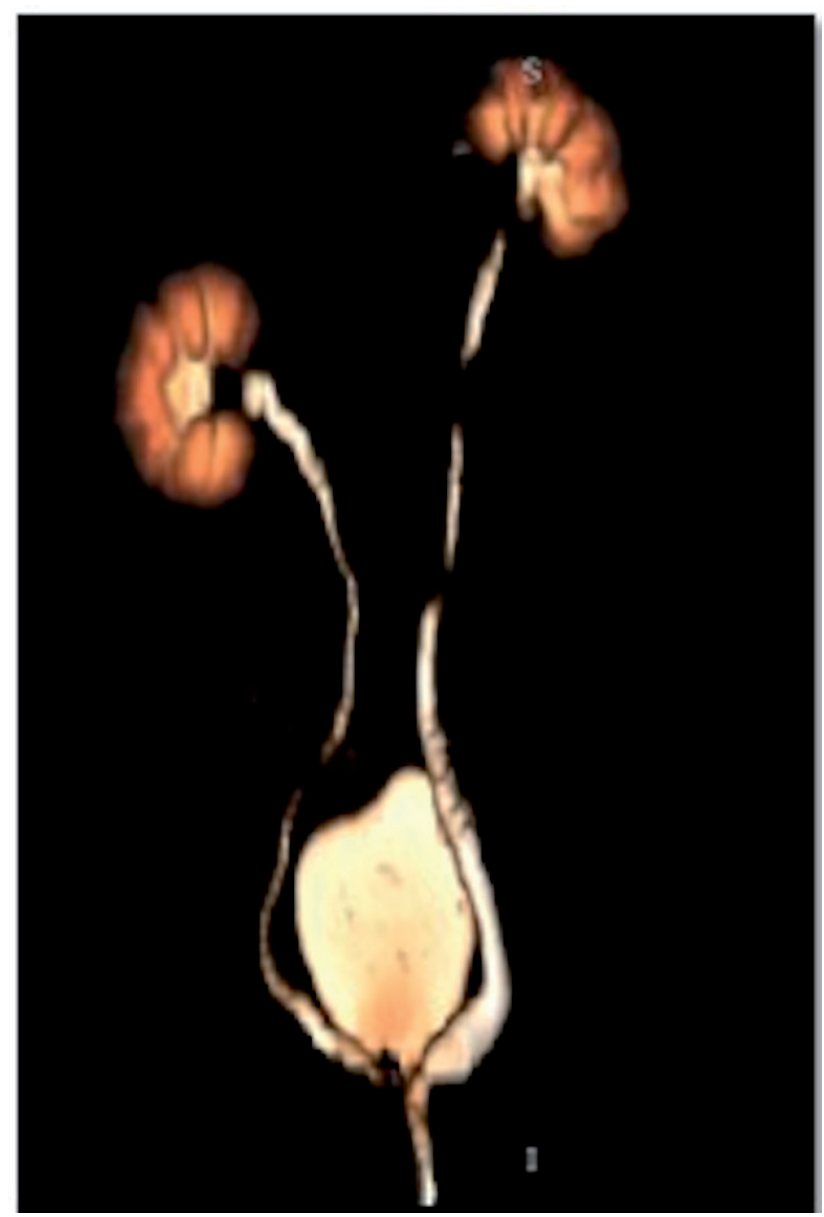

Figure 2. Uro-tomography image with a three-dimensional reconstruction showing the dilation of the right ureter in the insertion of the bladder trigone and the continued contrast leakage in the intramural path (orange arrow).
3-0 cistorraphy was performed in the first plane, with a Cushing suture pattern, and the second lembert plane. After omentopexy, laparorraphy was performed conventionally. The location of the double-J catheter was confirmed as ideal through abdominal radiography (Figure 4).

The patient underwent clinical evaluation and laboratory tests (complete blood count and serum creatinine concentration, urine test with bacteriological culture and susceptibility test) 2 weeks after the procedure and, subsequently, every 3 months. Ultrasonography of the urinary tract was performed every 2 months.

Three months after the second surgical procedure, the patient returned to the veterinary hospital with a history of dysuria and hematuria after estrus. Urinary infection by Staphylococcus sp. was diagnosed and treated with a combination of amoxicillin and clavulanate [Agemoxi CL ${ }^{\circledR 6}-22 \mathrm{mg} / \mathrm{kg}$ every 12 $\mathrm{h}$ for 30 days]. Ten months after placing the double $\mathrm{J}$ catheter, the patient returned to the veterinary hospital again with the same complaint of dysuria and hematuria. The signs of urinary incontinence had resolved after a second surgical procedure; however, the owner reported that urinary incontinence recurred together with phases of dysuria and hematuria. Hematological ultrasound and urinalysis tests were performed. The results of the bacterial culture of the urine sample were positive for Staphylococcus sp. The patient was treated with cephalexin [Celesporin ${ }^{\circledR 7}-30 \mathrm{mg} / \mathrm{kg}$ every $12 \mathrm{~h}$ for 60 days].

Fourteen months after surgical treatment, the patient had ambulatory tetraparesis, continuous spasms in the cutaneous muscle of the trunk in the cervical

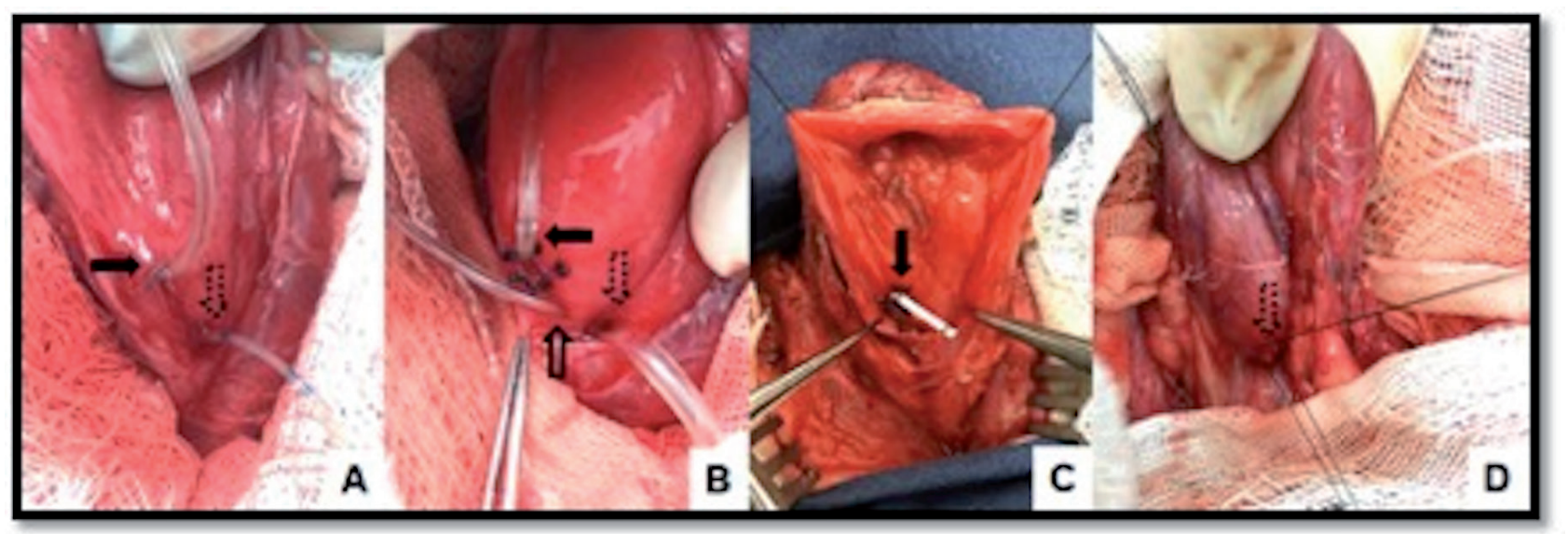

Figure 3. Transoperative images of the double $\mathrm{J}$ catheter placement in association with the neoureterostomy technique. A- Urinary bladder with created and probed ureteral ostium (black arrow) and intramural path identified with a $16 \mathrm{G}$ epidural catheter (dotted arrow). B- New urethral ostium sutured to the bladder mucosa (black arrow), intramural pathway with probe (empty arrow), and urethral probe 10 (dotted arrow). C- Final aspect of the new ureteral ostium after placement of a double $\mathrm{J}$ catheter with the distal end sectioned for better accommodation of the vesical lumen. D- Dorsal face of the bladder, with two separate simple points involving the intramural pathway, for posterior occlusion. 


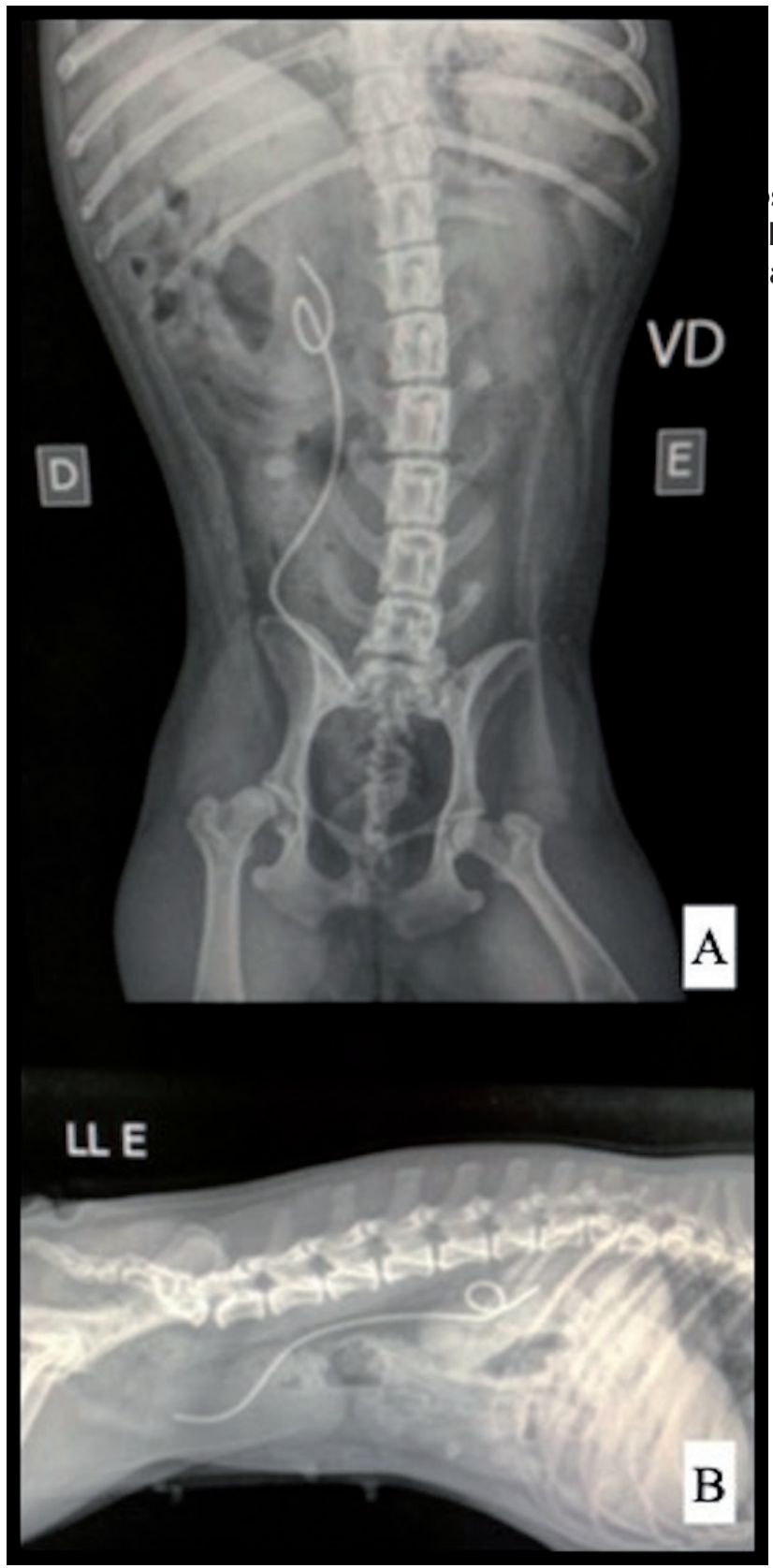

Figure 4. A- Ventrodorsal. B- Lateral radiographic views of the abdomen of a dog showing right-sided ureteral stent placement.

region, and pain on palpation in the transverse process of the 6th cervical vertebra. Radiographic evaluation revealed an increase in the $\mathrm{C} 6-\mathrm{C} 7$ intervertebral space caused by a decrease in the length of the C6 vertebra and the area of osteolysis at its caudal border (Figure 5 A \& C). Magnetic resonance imaging was performed and signs of discoespondilitis in the distal epiphysis of the 6th cervical vertebra were observed. Discoespondilitis secondary to urinary tract infection by Staphylococcus sp. was suspected [11]. As a result, the surgical procedure for removing the double $\mathrm{J}$ catheter, which could cause bacterial aggregation and resistance, was indicated and performed followed by treatment with cephalexin [Celesporin ${ }^{\circledR 7}$ - $30 \mathrm{mg} / \mathrm{kg}$ every $12 \mathrm{~h}$ for 6 months]. After 6 months of treatment, the radiographic examination showed improvement in the area of sta Jyniorysis at the L6 caudal border (Figure 5 B \& D). briel Luiz The patient had clinically improved neurological signs and there was no recurrence of cystitis or ureteral stenosis. This has remained stable (940 days after surgical correction of intramural ectopic ureter and placement of a double-J catheter and 510 days after removal of the double-J catheter).

\section{DISCUSSION}

Ectopic ureter is a rare congenital malformation in dogs. It can be classified as intramural or extramural depending on its anatomical position; the first presentation is the most commonly diagnosed in dogs, especially in females under one year of age [10]. Surgery is always indicated for correcting this anomaly and the prognosis varies with cases [8].

In this case, the patient had undergone a neoureterostomy and a closure of the intramural pathway of the ectopic ureter. Leakage of urine through the intramural path and dilation of the affected ureter (hydroureter) was observed on imaging tests. According to McLoughlin et al. [6], hydroureters commonly develop in the postoperative period due to the manipulation and edema; however, a return to normal diameter is expected within 4 to 6 weeks. In this case, the dilation of the ureter was observed on computed tomography and ultrasonography 12 months after the surgery. This was attributable to the stenosis of the ureteral ostium, which was later visible during the surgical reintervention.

The double $\mathrm{J}$ catheter was first described by Hepperlen et al. [3] and has been widely used in medicine [2,7]. Double J catheters (Double-J-stent) have been used in veterinary medicine to treat or prevent ureteral obstruction $[1,5,9]$. They have also been used to treat obstructive pyonephrosis [4] and iatrogenic trauma to the ureter [12]. It is most commonly introduced retrogradely through the ureter until the catheter coils in the renal pelvis and the other end is located in the bladder. In this way, the migration of the catheter to the urinary vesicle is avoided [7,12]. Due to the previous obstruction of the ureter ostium following the first surgical procedure, we used a double $\mathrm{J}$ catheter in the patient to prevent complications such as postoperative 


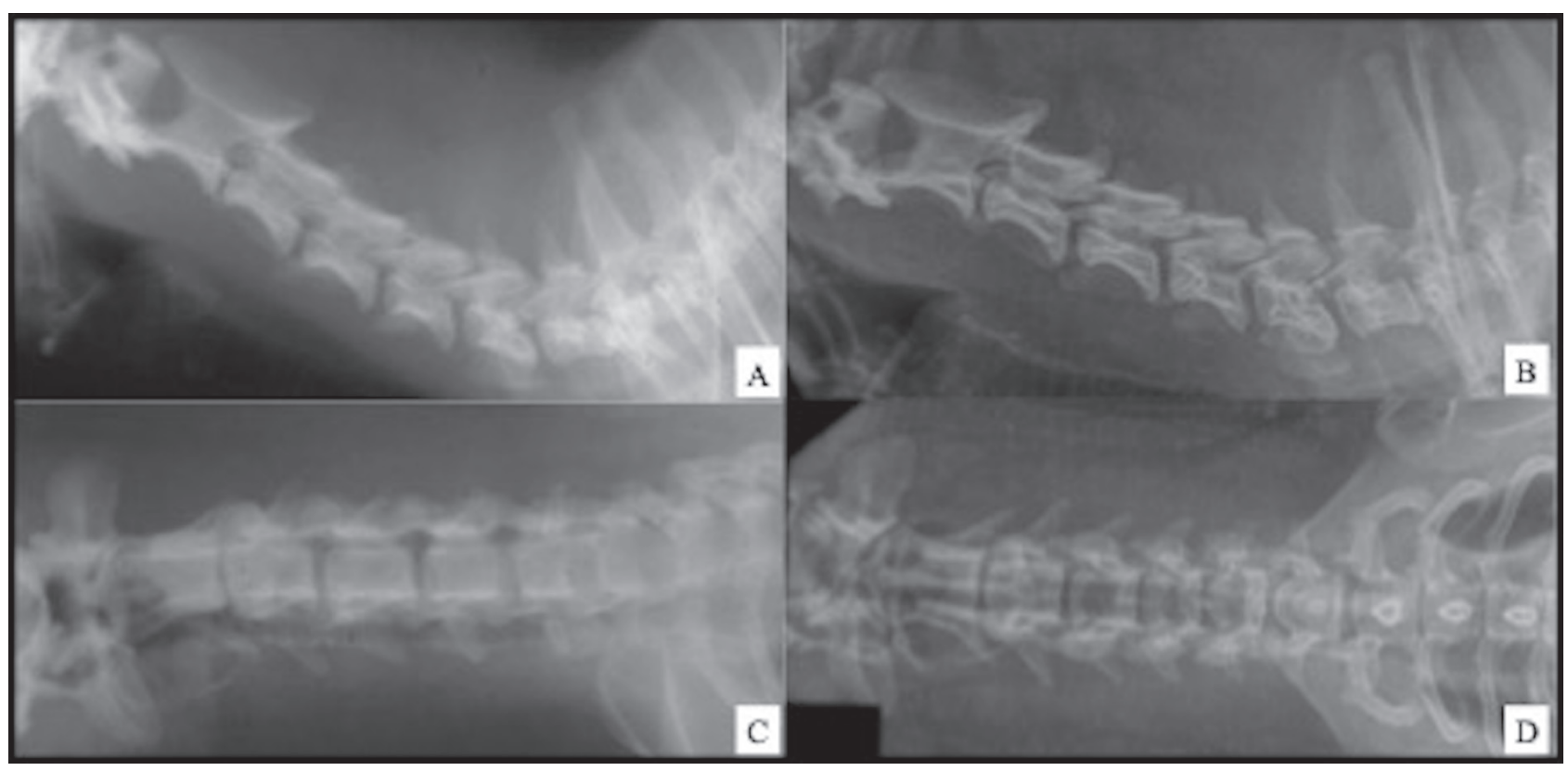

Figure 5. A- Radiographic images of discoespondilitis in dogs. B- Ventrodorsal radiography of the cervical spine showing osteolysis of the caudal epiphysis of the vertebral body (arrow) and comparative improvement of the radiographic aspect. C- Laterolateral radiography of the cervical spine showing concentric osteolysis of the body of the 6th cervical vertebra indicated by the arrow. D- Comparative improvement of the radiographic aspect.

obstructions due to ureteritis and ureteral constriction, and facilitate ureteral anastomosis [12]. Although there are minimally invasive techniques for placing this catheter in dogs, celiotomy with subsequent cystotomy is widely used because it is easy and safe [12].

After neoureterostomy, complications such as cystitis, recurrent urinary incontinence, and dysuria are common [8]. The same can occur after the placement of the double J catheter [1,4]. It has been reported that animals subjected to ureteral stent placement had a high incidence of dysuria and urinary tract infection, and less frequently, stent migration and occlusion [4]. In this case, no signs of occlusion or obstruction of the implant were identified, but there was a recurrence of urinary tract infections.

Culture tests of the patient's urine were carried out at various stages in the postoperative period and urinary tract infection was suspected. All results were positive for the growth of Staphylococcus sp. After 2 episodes and treatments of bacterial cystitis, the patient presented hyperesthesia on palpation of the sixth cervical vertebra, ambulatory tetraparesis, and muscle spasms in the cervical region. Discoespondilitis between the sixth and seventh cervical vertebrae was suspected and confirmed on radiography.

In the present study, the patient underwent a new urinalysis that confirmed the infection by Staphylococcus sp. after the diagnosis of discoespondilitis. The removal of the double $\mathrm{J}$ catheter was indicated and undertaken since the implant may have enabled the aggregation of bacteria and contributed to resistance to antimicrobial treatment. According to Hao et al. [2], complications similar to those found in this case are common in humans, and removal of the implant is often indicated when there is a decrease in quality of life or risk for the patient.

The study was limited by the confirmation of the diagnosis of discoespondilitis through incisional biopsy of the L6 vertebral body and the corresponding intervertebral disc (L6-L7), and histopathological examination. However, the improvement of neurological signs after treatment for the discoespondilitis, which was associated with improvement in the area of osteolysis at the L6 caudal border and the resolution of bacterial cystitis, support the diagnosis of discoespondilitis secondary to bacterial cystitis caused by Staphylococcus sp. After treatment for discoespondilitis, the patient was followed up for 12 months without recurrence.

The double $\mathrm{J}$ catheter may be useful for the correction of ectopic ureters and reduce complications related to the new ureteral opening; however, its long-term use can cause complications. A larger number of cases is required to determine the benefits and complications associated with its use.

\section{MANUFACTURERS}

${ }^{1}$ Medsonda - Indústria e Comércio De Produtos Hospitalares Descartáveis Ltda. Arapoti, PR, Brazil. 
${ }^{2}$ Smiths Medical do Brasil Produtos Hospitalares Ltda. São Paulo, SP, Brazil.

${ }^{3}$ Johnson \& Johnson do Brasil Indústria e Comércio de Produtos para Saúde Ltda. Vila Olímpia, SP, Brazil.

${ }^{4}$ Gadali Medical Comércio de Produtos Médico-Hospitalar Ltda. Campinas, SP, Brazil.

${ }^{5}$ Shalon Fios Cirúrgicos Ltda. São Luís de Montes Belos, GO, Brazil.
${ }^{6}$ Agener União Distribuidora de Medicamentos Ltda. São Paulo, SP, Brazil.

${ }^{7}$ Ouro Fino Saúde Animal Ltda. Cravinhos, SP, Brazil.

Declaration of interest. The authors report no conflicts of interest. The authors alone are responsible for the content and writing of this paper.

\section{REFERENCES}

1 Berent A.C., Weisse C., Beal M.W., Brown D.C., Tod K. \& Bagley D. 2011. Use of indwelling, double-pigtail stents for treatment of malignant ureteral obstruction in a dog: 12 cases (2006-2009). Journal of the American Veterinary Medical Association. 238(8): 1017-1025.

2 Hao P., Li W., Song C., Yan J., Song B. \& Li L. 2008. Clinical Evaluation of Double-pigtail Stent in Patients with Upper Urinary Tract Diseases: Report of 2685 Cases. Journal of Endocrinology. 22(1): 65-70.

3 Hepperlen T.W., Mardis H.K. \& Kammandel H. 1978. Self-retained Internal Ureteral Stents: a New Approach. The Journal of Urology. 119(6): 731-733.

4 Kuntz J.A., Berent A.C., Weisse C.W. \& Bagley D.H. 2015. Double pigtail ureteral stenting and renal pelvic lavage for renal-sparing treatment of obstructive pyonephrosis in dogs: 13 cases (2008-2012). Journal of the American Veterinary Medical Association. 246(2): 216-225.

5 Lam N.K., Berent A.C., Weisse C.K., Bryan C., Mackin A.J. \& Bagley D.H. 2012. Endoscopic placement of ureteral stents for treatment of congenital bilateral ureteral stenosis in a dog. Journal of the American Veterinary Medical Association. 240(8): 983-990.

6 Mcloughlin M.A. \& Chew D.J. 2000. Diagnosis and Surgical Management of Ectopic Ureters. Clinical Techniques in Small Animal Practice. 15(1): 17-24.

7 Mardis H.K., Hepperlen T.W. \& Kammandel H. 1979. Double Pigtail Ureteral Stent. Urology. 14(1): 23-25.

8 Noel S.M., Claeys S. \& Hamaide A.J. 2017. Surgical management of ectopic ureters in dogs: Clinical outcome and prognostic factors for long-term continence. Veterinary Surgery. 46(5): 631-641.

9 Pavia P.R., Berent A.C., Weisse C.W., Neiman D., Lamb K. \& Bagley D. 2018. Outcome of ureteral stent placement for treatment of benign ureteral obstruction in a dog: 44 cases (2010-2013). Journal of the American Veterinary Medical Association. 252(6): 721-731.

10 Reichler I.M., Specker C.E., Hubler M., Boos A., Haessig M. \& Arnold S. 2012. Ectopic Ureters in Dogs: Clinical Features, Surgical Techniques, and Outcome. Veterinary Surgery. 41(4): 515-522.

11 Tipold A. \& Stein V.M. 2010. Inflammatory Diseases of the Spine in Small Animals. Veterinary Clinics of North America: Small Animal Practice. 40(5): 871-879.

12 Wormser C., Clarke D.L. \& Aronson L.R. 2015. End-to-end ureteral anastomosis and double-pigtail ureteral stent placement for treatment of iatrogenic ureteral trauma in two dogs. Journal of the American Veterinary Medical Association. 247(1): 92-97. 